\title{
Relations between isoscalar charge form factors of two- and three-nucleon systems
}

\author{
H. Henning, ${ }^{1}$ J. Adam, Jr., ${ }^{2,3}$ P. U. Sauer, ${ }^{1}$ and A. Stadler ${ }^{1,4}$ \\ ${ }^{1}$ Institute for Theoretical Physics, University Hannover, D-30167 Hannover, Germany \\ ${ }^{2}$ Nuclear Physics Institute, Czech Academy of Sciences, CZ-25068 Řežnear Prague, Czech Republic \\ ${ }^{3}$ Institute for Nuclear Physics, University Mainz, D-55099 Mainz, Germany \\ ${ }^{4}$ Department of Physics, College of William and Mary, Williamsburg, Virginia 23185 \\ (Received 16 August 1993; revised manuscript received 2 December 1994)
}

\begin{abstract}
Theoretical predictions for the deuteron and the isoscalar trinucleon charge form factors are compared. Correlations between them are found. Linear relations hold for the position of the diffraction minimum and for the position and height of the secondary maximum. The linear relation for the diffraction minimum does not pass through the experimental data.

PACS number(s): 25.30.Bf, 21.45+v, 25.10. $+\mathrm{s}, 27.10 .+\mathrm{h}$
\end{abstract}

The measurement [1] of the deuteron tensor polarization in elastic electron scattering allows the separation of the deuteron charge form factor into its monopole and quadrupole parts. The electromagnetic (e.m.) form factors of the trinucleon isodoublet ${ }^{3} \mathrm{He}$ and ${ }^{3} \mathrm{H}$ have been measured in detail [2] and their separation into isoscalar and isovector components, respectively, was done [3] by assuming perfect isospin symmetry. Thus, the e.m. properties of the isosinglet deuteron and the isoscalar trinucleon properties can now be compared in a clean way. This paper carries out that comparison for charge monopole form factors called here charge form factors (CFFs) without multipole specification for short.

Figure 1 shows the deuteron and the isoscalar trinucleon CFFs as extracted from the experimental data [1,3]; we choose to compare them with predictions of the Paris [4] and the Bonn B [5] potentials. Results for a variety of other potentials will be discussed later. The charge operator contributes to those CFFs only by its isoscalar part. Its one-nucleon part is parametrized in terms of Dirac and Pauli proton and neutron form factors according to Refs. $[6,7]$; it alone survives in nonrelativistic impulse approximation (NRIA). In the deuteron, NRIA is rather successful for the Paris potential, though unsatisfactory for the Bonn B potential. In the three-nucleon system, NRIA fails for both potentials. We observe that the charge operator corrections which lead to an improved description of the trinucleon CFF for both potentials yield a poor deuteron description, even destroying the fair agreement for the deuteron achieved in NRIA by the Paris potential. The quadrupole form factor is also shown in Fig. 1; it is only mildly influenced by them.

For the calculations of Fig. 1 we use a charge operator which contains one- and two-nucleon corrections of relativistic order. The two-nucleon corrections are based on onemeson exchange, i.e., on meson exchange up to the second order in the meson-nucleon coupling constants. In general, the mesons contribute through the meson-diagonal contact, meson-current, and Born processes. In the present calculation the isoscalar contributions of the pseudoscalar $\pi$ - and $\eta$-, the scalar $\sigma$ - and $\delta$-, and the vector $\rho$ - and $\omega$-mesons of the Bonn potentials are used in the form given by the extended $S$-matrix method $[8,9]$. Among the meson nondiagonal contributions only the $\rho \pi \gamma$ process is included as given in Ref. [10] which is the only important one in the region of momentum transfer considered. The resulting charge operator satisfies, together with the spatial current operator $\mathbf{j}(\mathbf{Q})$, the continuity equation, i.e.,

$$
\begin{gathered}
\mathbf{Q} \cdot \mathbf{j}^{[1]}(\mathbf{Q})=\left[k, \rho^{[1]}(\mathbf{Q})\right], \\
\mathbf{Q} \cdot \mathbf{j}^{[2]}(\mathbf{Q})=\left[k, \rho^{[2]}(\mathbf{Q})\right]+\left[v, \rho^{[1]}(\mathbf{Q})\right] .
\end{gathered}
$$

The one- and two-nucleon character of the operators is indicated by superscripts 1 and 2 , respectively; $k$ is the kinetic energy operator with relativistic energies, $v$ the two-nucleon potential, and $\mathbf{Q}$ the momentum transfer to the nucleus; the commutator $\left[v, \rho^{[2]}(\mathbf{Q})\right]$ has two-nucleon contributions, but it does not arise in Eq. (1), since it is of fourth order in the meson-nucleon coupling constants.

The extended $S$-matrix method, as well as alternative techniques, do not yield unique potential and charge and current operators, but a family of unitary equivalent ones, characterized by the parameters $(\tilde{\mu}, \nu)$. Once a meson-exchange potential is defined, the parameters $(\tilde{\mu}, \nu)$ are uniquely determined. Among those parameters, $\tilde{\mu}$ generalizes [9] the parameter $\mu$ which was introduced by Friar [11] and which describes the mixture of pseudoscalar and pseudovector coupling for pseudoscalar mesons with the nucleon. In contrast to $\mu$, the parameter $\tilde{\mu}$ also contains the arbitrariness in the treatment of the energy transfer at the pion-nucleon vertex. The parameter $\nu$ is connected with meson retardation; it describes the off-energy shell arbitrariness in the meson propagator; the choice $\nu=1 / 2$ makes its dependence on the energy transfer disappear in the potential. The charge and current operators are expanded in powers of $(v / c), v$ being a characteristic nucleon velocity. The present calculation employs the charge operator up to order $(v / c)^{2}$. The resulting isoscalar charge operator is local.

The individual contributions to the CFFs arising from the different terms in the charge operator are discussed in detail elsewhere [12]; the pion contributions are dominant. Their effects on the deuteron and trinucleon CFFs are strikingly similar, suggesting relations which we now investigate. As a function of three-momentum transfer $\mathbf{Q}$ in the Breit frame, the CFFs are qualitatively characterized (i) by their slope at zero momentum transfer, i.e., by their rms charge radius, (ii) by the position $\mathbf{Q}_{\min }^{2}$ of their first diffraction minimum, (iii) by the position $\mathbf{Q}_{\max }^{2}$, and (iv) by the height $F\left(\mathbf{Q}_{\max }^{2}\right)$ of their secondary maximum. The rms charge radius is mainly deter- 

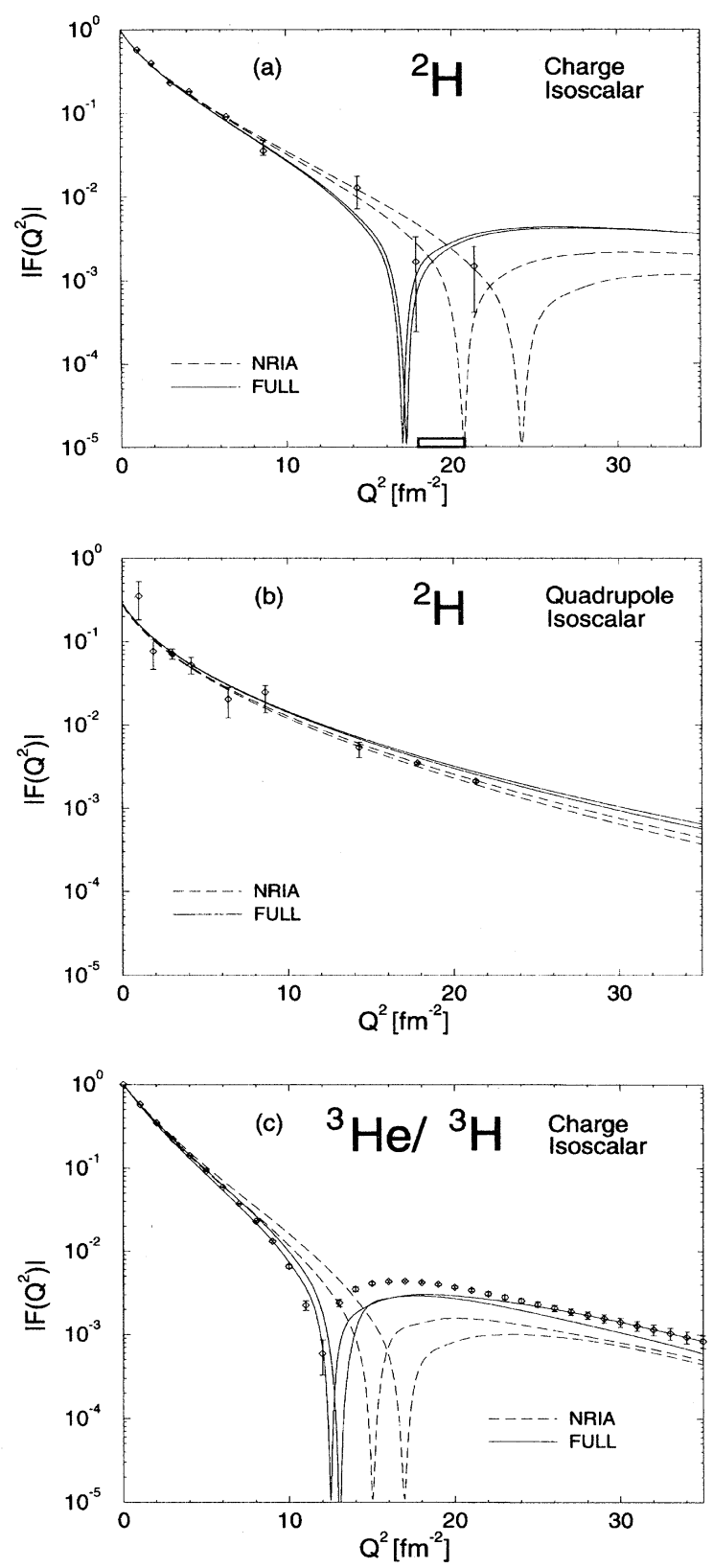

FIG. 1. Deuteron monopole (a), quadrupole (b), and normalized isoscalar trinucleon (c) CFFs as functions of three-momentum transfer $\mathbf{Q}^{2}$. The theoretical predictions refer to the Paris and Bonn $B$ potentials. Predictions derived in NRIA are shown as dashed curves, those derived from a full calculation (FULL) with all charge corrections up to order $(v / c)^{2}$ included as solid curves. The pair of curves with smaller (larger) minimum positions in the deuteron monopole and in the isoscalar trinucleon CFFs and with lower (larger) values in the deuteron quarupole CFF belong to the Paris (Bonn B) potential. The experimental data are taken from Refs. $[1,3]$. The experimentally determined position of the deuteron diffraction minimum is indicated by the horizontal box.

mined by the asymptotics of the wave function, e.g., the binding energy, which in the case of the deuteron is a fit parameter. Thus, we shall try to find relations for the characterizing properties (ii)-(iv) of the CFFs only. We find them between predictions for different realistic two-nucleon potentials. They are novel, Ref. [13] only gives a qualitative discussion of two- and three-nucleon CFFs with respect to their possible relations.

Six two-nucleon potentials are used. The parameters $(\tilde{\mu}, \nu)$, governing their respective off-shell extension, i.e., the amount of chosen nonlocalities in the potentials, and determining their consistent charge and current operators, are given. They are the Paris $(0,1 / 2)$ potential, based on the dispersion-theoretic approach, the purely phenomenological Reid soft-core RSC $(-1,1 / 2)$ potential [14], and the Bonn $(-1,1 / 2)$ one-boson exchange potentials OBEPQ [15], A, B, and $\mathrm{C}[5]$. The arguments for the choice of $(\tilde{\mu}, \nu)$ are given in Ref. [9]. The results for the hadronic triton properties derived from the Paris, RSC, and Bonn OBEPQ potentials are given in Refs. [16,17]. Our results for the Bonn A, B, and C potentials have not been published, but they are, by and large, consistent with those of Ref. [5].

The calculation of the CFFs is carried out in momentum space. Against the tradition of the authors [17-19], the trinucleon calculations are purely nucleonic ones without $\Delta$-isobar degrees of freedom: Single $\Delta$-isobar excitation affects the trinucleon isoscalar CFF only slightly. The meson parameters used in the charge operator are chosen for the Bonn potentials as the Bonn potentials define them. In contrast, the present calculations with the Paris and RSC potentials employ the $\pi$ - and $\rho$-meson parameters of Ref. [19], whereas the parameters of the other mesons are taken from the Bonn OBEPQ potential.

We find remarkable linear relations between corresponding characteristic features of the deuteron $d$ and the normalized isoscalar trinucleon $t$ CFFs $F^{[d]}$ and $F^{[t, I S]}$, i.e., $\mathbf{Q}^{[t] 2}=a \mathbf{Q}^{[d] 2}+b$ for the minimum and maximum positions and $F^{[t, I S]}\left(\mathbf{Q}_{\max }^{[t] 2}\right)=c F^{[d]}\left(\mathbf{Q}_{\max }^{[d] 2}\right)$ for the height of the secondary maximum. The three relations are displayed in Fig. 2. The relations hold for results obtained for a variety of twonucleon potentials; they hold in NRIA and with the inclusion of one- and two-nucleon charge corrections of relativistic order $(v / c)^{2}$. The common slope $a$ of the linear relations is $a=0.60$ for the position of the diffraction minimum and the secondary maximum, the common intersect is $b=2.43$. In the relation for the height of the secondary maximum we find $c=0.78$. The relations appear independent from the details of the considered charge model: A charge operator, inconsistent with the underlying potential, e.g., in choice of values $(\tilde{\mu}, \nu)$ or coupling constants and cut-off parameters, creates only small deviations of the prediction from the linear relations, as long as the same operators are used for both systems, changing at the same time the individual CFFs considerably, allowing even to bring one of them close to experimental data.

The relations found are conceptually interesting. We do not have an explanation for them, but make the following three observations.

(1) Assuming the validity of the quasideuteron model for the triton wave function to be precise and the triton wave function symmetric under the interchange of the weighted Jacobi momenta $p$ and $(\sqrt{3} / 2) q$, ( $p$ being the relative mo 

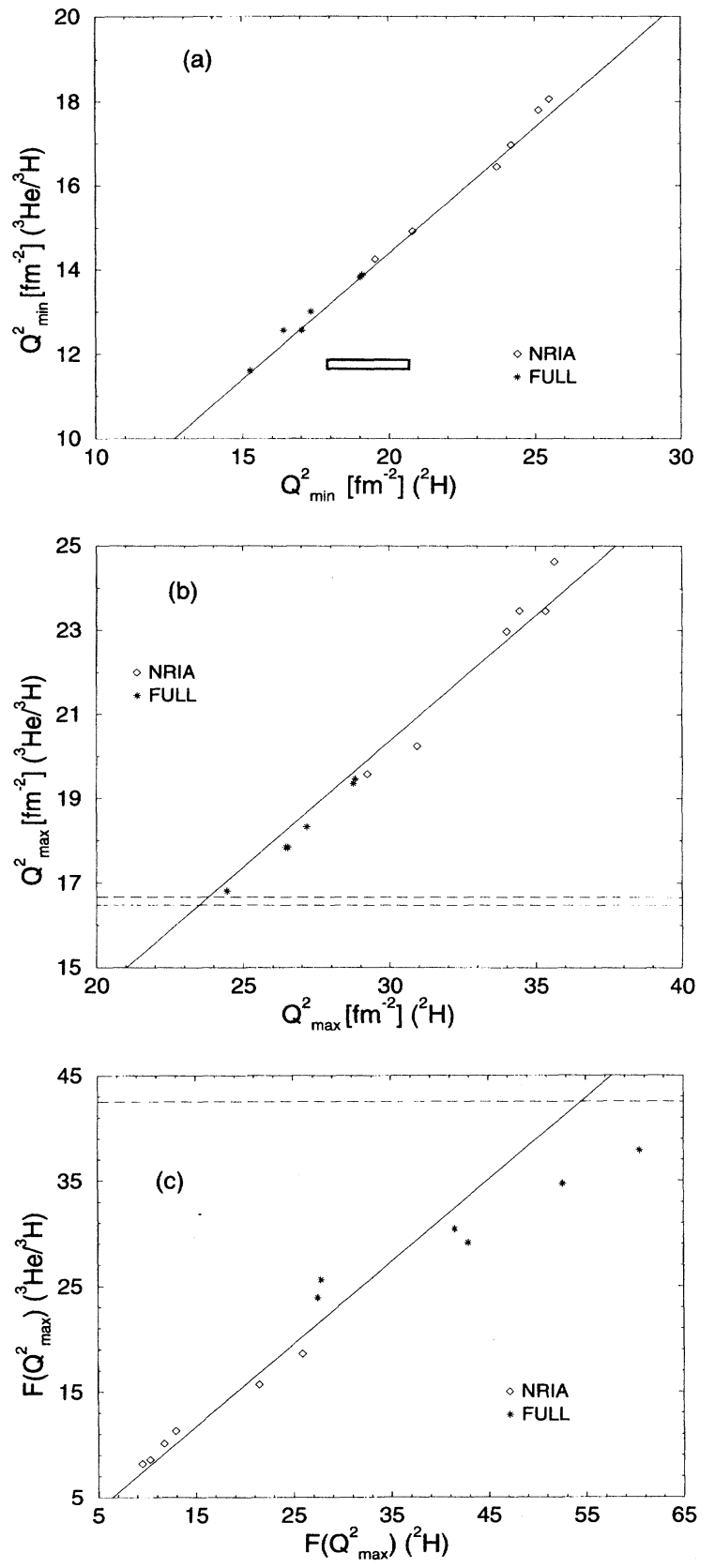

mentum of a nucleon pair, $q$ between the pair c.m. and the spectator nucleon), Ref. [21] also notices relations between deuteron and trinucleon CFFs in NRIA.

(2) The quasideuteron model is not very well founded. Reference [22] supports it qualitatively, but Ref. [23] demonstrates deficiencies of the quasideuteron assumption (1). Nevertheless, the employed trinucleon wave functions show approximate symmetry on the interchange of the weighted Jacobi momenta $p$ and $(\sqrt{3} / 2) q$ in the components with pair orbital angular momentum zero, most important for the trinucleon CFFs. In fact, that approximate symmetry underlies the famous relation [24] between the ${ }^{3} \mathrm{He}-{ }^{3} \mathrm{H}$ charge asymmetry and the individual trinucleon CFFs.

(3) The linear relations, for which Fig. 2 gives an ex-
FIG. 2. Position $\mathbf{Q}_{\min }^{2}$ of the diffraction minimum, position $\mathbf{Q}_{\max }^{2}$ and height $F\left(\mathbf{Q}_{\max }^{2}\right)$ of the secondary maximum in the deuteron and in the isoscalar trinucleon CFFs. The results for six potentials are shown, each distinguished for nonrelativistic impulse approximation (NRIA) and for the full calculation (FULL) with all charge corrections included up to order $(v / c)^{2}$. The results of the full calculation belong to the potentials RSC, Bonn C, Paris, Bonn B, Bonn OBEPQ, Bonn A from lower left to upper right in parts (a) and (b) of the plot; in the NRIA results the order of entries is interchanged between the Bonn $\mathrm{C}$ and Paris potentials; those sequences are reversed in part (c). The straight lines are least-square fits simultaneously for minimum and maximum positions with the slope $a=0.60$ and $b=2.43$ for (a) and (b) and the slope $c=0.78$ for (c). The results of Ref. [20] are in between our RSC and Paris results and seem not to deviate from the found correlations. The experimental values for the diffraction minima are taken from Refs. $[1,3]$ and indicated by the box, the secondary maximum is experimentally only seen in the trinucleon charge form factor; the experimental trinucleon value for position and height is, respectively, given by two horizontal lines, indicating its upper and lower values; in (c) the upper value coincides with the frame. If individual linear relations were allowed for the positions of the diffraction minimum and the secondary maximum and if the linear relation for the height of the secondary maximum were not forced to go through the origin in anticipation of Eq. (2), the calculated results would follow unconstrained linear relations much better. ample, allow the CFFs to be completely related at larger momentum transfers according to

$$
F_{\mathrm{est}}^{[d]}\left(\mathbf{Q}^{2}\right)=c^{-1} F^{[t, I S]}\left(a \mathbf{Q}^{2}+b\right) .
$$

Figure 3 demonstrates for the CFFs resulting from the Bonn $B$ potential how well relation (2) is realized. The parameters $a, b$, and $c$ could be optimized by making the deuteron CFF $F_{\text {est }}^{[d]}\left(\mathbf{Q}^{2}\right)$, estimated according to Eq. (2) coincide with the calculated deuteron CFF $F^{[d]}\left(\mathbf{Q}^{2}\right)$ precisely at the position of the diffraction minimum, at the position and in the height of the secondary maximum; the optimized parameters cluster around those given above. Thus, $F_{\text {est }}^{[d]}\left(\mathbf{Q}^{2}\right) \simeq F^{[d]}\left(\mathbf{Q}^{2}\right)$. The deviation decreases with increasing momentum transfer $\mathbf{Q}^{2}$, it is at most $33 \%$ for $\mathbf{Q}^{2} \simeq 9 \mathrm{fm}^{-2}$ and is less than $5 \%$ 


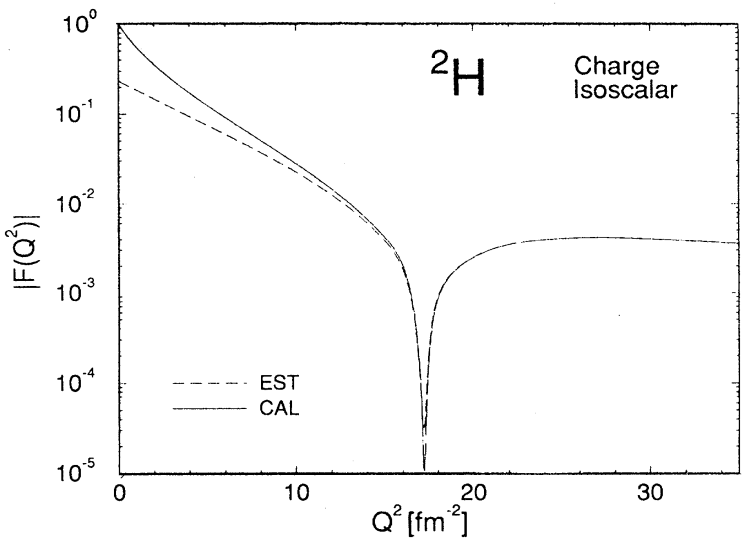

FIG. 3. Deuteron CFF $F^{[d]}\left(\mathbf{Q}^{2}\right)$ for the Bonn B potential including all charge operator corrections of order $(v / c)^{2}$. The result of a full calculation, shown as solid curve (CAL) and identical to the one of Fig. 1(a), is compared to the deuteron CFF $F_{\text {est }}^{[d]}\left(Q^{2}\right)$, estimated according to Eq. (2) from the calculated isoscalar trinucleon CFF; the estimated deuteron CFF is shown as dashed curve (EST). The parameters employed in Eq. (2) for this figure are optimized ones with $a=0.54, b=3.63$, and $c=0.73$.

beyond the diffraction minimum. The quasideuteron model yields a relation between deuteron and trinucleon CFFs corresponding to Eq. (2); the parameters are, however, with $a=3 / 4, b=0.00$, and $c=1.24$ rather different [the original $c=1.86$ [21] has been converted according to our normalization $\left.F^{[t, I S]}(0)=1\right]$. The value of $c$ results from a fit to the experimental CFFs at momentum transfers $\mathbf{Q}^{2}$ below the minimum where relation (2) is rather poor.

The experimental value for the minimum position of the isoscalar CFF is $\mathbf{Q}^{2}=19.27+1.43-1.38 \mathrm{fm}^{-2}$ for the deuteron [1] and $\mathbf{Q}^{2}=11.75 \pm 0.11 \mathrm{fm}^{-2}$ for the trinucleon [3]. The linear relation for the diffraction minimum $\mathbf{Q}_{\min }^{2}$ passes the experimental data and their present error bars by a substantial margin. That fact is another illustration for our earlier observation [25] that the existing range of realistic nonrelativistic two-nucleon potentials together with the present understanding of meson-exchange currents is unable to account simultaneously for the e.m. properties of the two- and threenucleon systems. If persisting, that observation has serious implications. What are possible objections against the calculations on which the found relations are based? We list five.

(1) Despite the included charge operator corrections of relativistic order the present calculation cannot match the consistency of the covariant deuteron treatment of Ref. [26]. However, a fully covariant and realistic description of the three-nucleon system does not exist yet. Thus, covariant results for the deuteron and trinucleon CFFs cannot be compared in the same way as this paper does for noncovariant results. Moreover, a relativistic calculation of trinucleon CFFs [27] with schematic interactions shows a qualitative similarity to nonrelativistic results.

(2) The charge operator used here is expanded up to order $(v / c)^{2}$. Its neglected part of higher order in $(v / c)$ is not expected to change the results found for the considered momentum transfers.

(3) Relativistic boost corrections [28] are included for the deuteron. They are kinematic and without them the discrepancy encountered in Fig. 2 would even be larger. The boost

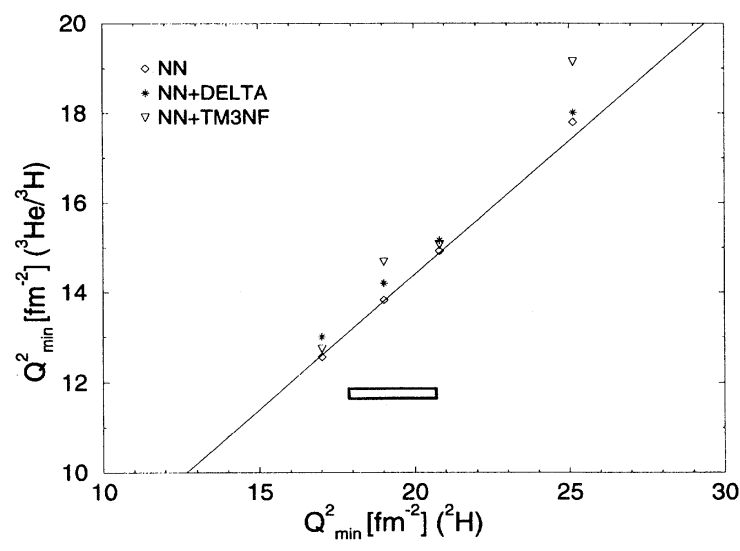

FIG. 4. Figure 2(a) is redrawn, keeping the fitted straight line and the entries for the two-nucleon (NN) Paris and Bonn OBEPQ potentials. In addition results are indicated for both potentials extended according to Refs. [17-19] by single $\Delta$-isobar excitation (DELTA) and with the irreducible pion- and rho-exchange TucsonMelbourne three-nucleon force added (TM3NF). The shown results are obtained in NRIA and in a full calculation with all charge corrections up to order $(v / c)^{2}$ included. They belong from the lower left to the upper right first to the full Paris and Bonn OBEPQ potentials and then to both again in NRIA.

corrections become more complicated and interaction dependent in the three-nucleon system. They have only been calculated schematically [29]. In general, they are believed to be smaller there compared with the deuteron.

(4) The trinucleon properties are affected $[18,30]$ by $\Delta$-isobar excitation. It yields an effective three-nucleon force and an e.m. exchange current. The three-nucleon force increases the trinucleon binding energy and therefore shifts the diffraction minimum of the CFF toward larger momentum transfers enhancing the disagreement between experimental and theoretical values. Most $\Delta$-isobar exchange currents are of an isovector nature. Predictions based on single $\Delta$-isobar excitation preserve the correlations of Fig. 2 as Fig. 4 proves. Effects of double $\Delta$-isobar excitations on the deuteron e.m. properties are small [31]. Corresponding ones on the threenucleon bound state have not been calculated yet. In both systems they will move the diffraction minima simultaneously to larger values. A possible shift of the observed straight line of results is expected to be minute.

(5) The quoted treatment of single $\Delta$-isobar excitation is technically proper. However, the resulting three-nucleon force is incomplete in its physics content. A method for unifying the $\Delta$-isobar approach to the three-nucleon force and its description by an irreducible operator is given in Ref. [32], but has not been applied yet to the e.m. trinucleon properties. Once the full Tucson-Melbourne three-nucleon force [33] is added as an irreducible three-nucleon operator to two-nucleon potentials, i.e., to the RSC, Paris and Bonn OBEPQ, the results slide along the straight lines in all relations, as Fig. 4 shows for the diffraction minimum. The results do so irrespectively, if the trinucleon wave functions are derived [34] with the pure pion or with the combined pionrho exchange three-nucleon force, and irrespectively, if the CFFs are calculated in NRIA or with two-nucleon exchange corrections added. We observe that the linear relations are only broken for the Bonn OBEPQ potential in which the employed three-nucleon force overbinds the triton heavily by 
1.15 MeV. We expect that scaled trinucleon calculations which fine-tune [35] the three-nucleon force to yield the computed triton binding energy consistent with its experimental value, will even better respect the observed linear relations. Three-nucleon exchange corrections of the charge operator fully consistent with an irreducible three-nucleon force of pion range have been given [36], some have been calculated in Ref. [37] and found negligible, except those with $\Delta$-isobar excitation.

This paper claims that the widely used "standard" model of meson-exchange currents, based on a one-meson exchange picture and $v / c$ expansions, is unable to account simultaneously for the e.m. properties of the two- and threenucleon systems. We emphasize again that one is left with considerable freedom in the choice of the two-nucleon potential, in the parametrization of the e.m. nucleon form factors, and in some other parameters, such as coupling constants and cut-off parameters, which are especially for heavier mesons only poorly determined. Using this freedom one can easily reach agreement with the data when only one nucleus is considered at a time. The same is also expected for the four-nucleon system and other light nuclei. Thus, one should be very careful in interpreting the results of calculations concerning only one nucleus. Clearly, additional studies are needed to clarify the physical reasons for the failure of the standard MEC model, to determine more precisely its range of applicability, and possibly to improve its extension into the momentum transfer region considered in this work. Only then can one consider the e.m. properties of more complex systems with some confidence.

Our conclusions rely on the experimental deuteron CFF as derived from the measured tensor polarization [1]. The deuteron polarization experiment is an admirable technical achievement, but additional data with decreased error bars in the range of momentum transfers already measured and additional data at larger momentum transfers would be highly welcome for an improved determination of the minimum position in the deuteron monopole CFF and for a first determination of the position and height of its secondary maximum.

The work was funded by the Deutsche Forschungsgemeinschaft (DFG) under Contract Nos. Sa 247/7-2, Sa 247/ 7-3, Sa 247/9-4, and 436 CSR-111/4/90, and by the DOE under Grant No. DE-FG05-88ER40435. During part of the work J.A. was fellow of the Humboldt Foundation. The calculations were performed at Regionales Rechenzentrum für Niedersachsen (RRZN), Hannover, at Rechenzentrum Kiel, at Continuous Electron Beam Accelerator Facility (CEBAF), and at National Energy Research Supercomputer Center (NERSC), Livermore.
[1] I. The et al., Phys. Rev. Lett. 67, 173 (1991).

[2] B. Frois and S. K. Platchkov, in Modern Topics in Electron Scattering, edited by B. Frois and I. Sick (World Scientific, Singapore, 1991), p. 320 and references there.

[3] A. Amroun et al., Phys. Rev. Lett. 69, 253 (1992).

[4] M. Lacombe et al., Phys. Rev. C 21, 861 (1980).

[5] R. Machleidt, Adv. Nucl. Phys. 19, 189 (1989).

[6] G. G. Simon et al., Nucl. Phys. A333, 381 (1980).

[7] S. Galster et al., Nucl. Phys. B32, 221 (1971).

[8] J. Adam, Jr., E. Truhlík, and D. Adamová, Nucl. Phys. A492, 556 (1989).

[9] J. Adam, Jr., H. Göller, and H. Arenhövel, Phys. Rev. C 48, 370 (1993).

[10] I. S. Towner, Phys. Rep. 155, 263 (1987).

[11] J. L. Friar, Ann. Phys. (N.Y.) 104, 380 (1977).

[12] H. Henning, J. Adam, Jr., and P. U. Sauer (in preparation).

[13] M. I. Haftel and W. M. Kloet, Phys. Rev. C 15, 404 (1977).

[14] R. V. Reid, Ann. Phys. (N.Y.) 50, 411 (1968).

[15] R. Machleidt, K. Holinde, and Ch. Elster, Phys. Rep. 149, 1 (1987).

[16] Ch. Hajduk and P. U. Sauer, Nucl. Phys. A369, 321 (1981); A. Stadler, W. Glöckle, and P. U. Sauer, Phys. Rev. C 44, 2319 (1991).

[17] H. Henning, P. U. Sauer, and W. Theis, Nucl. Phys. A537, 367 (1992).

[18] Ch. Hajduk, P. U. Sauer, and W. Strueve, Nucl. Phys. A405, 581 (1983).

[19] W. Strueve, Ch. Hajduk, P. U. Sauer, and W. Theis, Nucl. Phys. A465, 651 (1987).

[20] R. Schiavilla, V. R. Pandharipande, and D. O. Riska, Phys. Rev. C 41, 309 (1990); R. Schiavilla and D. O. Riska, ibid. 43, 437 (1991).
[21] B. Gangopadhyay and J. S. Levinger, J. Phys. G 18, 1933 (1992); 19, 1417 (1993).

[22] E. Hadjimichael, S. N. Yang, and G. E. Brown, Phys. Lett. 39B, 594 (1972).

[23] Ch. Hajduk, A. M. Green, and M. E. Sainio, Nucl. Phys. A337, 13 (1980).

[24] M. Fabre de la Ripelle, Fizika 4, 1 (1972); J. L. Friar, Nucl. Phys. A156, 43 (1970); R. A. Brandenburg, S. A. Coon, and P. U. Sauer, ibid. A294, 305 (1978).

[25] H. Henning, J. Adam, Jr., and P. U. Sauer, Few Body Syst. Suppl. 5, 133 (1992).

[26] E. Hummel and J. A. Tjon, Phys. Rev. C 42, 423 (1990), and references there.

[27] G. Rupp and J. A. Tjon, Phys. Rev. C 45, 2133 (1992).

[28] J. L. Friar, in Mesons in Nuclei, edited by M. Rho and D. H. Williamson (North-Holland, Amsterdam, 1979), p. 596; Phys. Rev. C 22, 796 (1980).

[29] J. L. Friar, Ann. Phys. (N.Y.) 81, 332 (1973).

[30] A. Picklesimer, R. A. Rice, and R. Brandenburg, Phys. Rev. C 44, 1359 (1991).

[31] R. Dymarz and F. C. Khanna, Nucl. Phys. A516, 549 (1990), and references there.

[32] A. Stadler and P. U. Sauer, Phys. Rev. C 46, 64 (1992).

[33] S. A. Coon and M. T. Peña, Phys. Rev. C 48, 2559 (1993), and references there.

[34] A. Stadler, J. Adam, Jr., H. Henning, and P. U. Sauer, Phys. Rev. C 51, 2896 (1995).

[35] J. L. Friar, Few Body Syst. Suppl. 1, 94 (1986); T. Sasakawa, ibid. 1, 104 (1986).

[36] S. A. Coon and J. L. Friar, Phys. Rev. C 34, 1060 (1986).

[37] D. O. Riska and M. Radomski, Phys. Rev. C 16, 2105 (1977). 